\title{
BMJ Open Evaluation of the association between sleep duration and tooth loss among Korean adults: data from the Korean National Health and Nutrition Examination Survey (KNHANES 2012-2014)
}

Kyungdo Han, ${ }^{1}$ Jun-Beom Park ${ }^{2}$

To cite: Han K, Park J-B. Evaluation of the association between sleep duration and tooth loss among Korean adults: data from the Korean National Health and Nutrition Examination Survey (KNHANES 2012-2014). BMJ Open 2018;8:e018383. doi:10.1136/ bmjopen-2017-018383

- Prepublication history for this paper is available online. To view these files, please visit the journal online (http://dx.doi. org/10.1136/bmjopen-2017018383).

Received 25 June 2017 Revised 27 March 2018 Accepted 18 April 2018

Check for updates

${ }^{1}$ Department of Biostatistics, College of Medicine, Catholic University of Korea, Seoul, Republic of Korea

${ }^{2}$ Department of Periodontics, College of Medicine, Catholic University of Korea, Seoul, Republic of Korea

Correspondence to Dr Jun-Beom Park; jbassoonis@yahoo.co.kr

\section{ABSTRACT}

Objectives This study assessed the association between sleep duration and tooth loss using nationally representative data. In this study, a cross-sectional analysis was performed using multivariable logistic regression analysis models.

Setting The present study analysed data from the Korean National Health and Nutrition Examination Survey between 2012 and 2014.

Participants A total of 14675 respondents over 19 years old without missing values were included in this study.

Exposure and primary outcome measures Sleep duration and tooth loss.

Results Participants with a sleep duration of 6-8hours showed the lowest prevalence of diabetes mellitus, hypertension, metabolic syndrome, periodontitis and meeting the waist circumference criteria for metabolic syndrome. Adjusted OR and their $95 \% \mathrm{Cl}$ of male individuals with fewer than 25 natural teeth were 1.426 (1.113 to 1.827), 1.290 (1.074 to 1.548), 0.988 (0.853 to 1.145), 1 (reference), 1.058 (0.907 to 1.235 ) and 1.620 (1.287 to 2.038) for sleep duration of 4 hours or less, 5 hours, 6 hours, 7 hours, 8 hours and 9 hours or more, respectively $(p<0.05)$, after adjustments for age, sex, smoking, drinking, walking, frequency of tooth brushing per day, body mass index and periodontitis.

Conclusions Our findings showed the U-shaped association between sleep duration and tooth loss was suggested by multiple logistic regression analyses after adjusting for confounding factors. Moreover, subgroup analyses showed that short and long sleep duration were associated with greater tooth loss in participants without diabetes mellitus, those without hypertension and those without metabolic syndrome.

\section{INTRODUCTION}

Previous studies have shown that sleep duration is associated with systemic diseases. ${ }^{1}$ Sleep was reported to be associated with cardiovascular function, including physiological conditions. ${ }^{2}$ The quantity of sleep is reported
Strengths and limitations of this study

- This study used nationally representative data with multistage clustered probability design, and the dental examinations were performed by trained dentists.

- The design of this study is cross-sectional, and the causal direction cannot be evaluated.

- The duration of sleep was evaluated via interviews with the participants, and there may be recall bias.

to be associated with the incidence of type 2 diabetes mellitus. ${ }^{3}$ Previous research showed that higher prevalence of obesity was associated with short sleep duration. ${ }^{4}$ In addition, prevalence of depression was independently associated with poor sleep. ${ }^{5}$ The previous results showed that increased OR of being obese were noted in individuals with $\leq 5$ hours of sleep (OR of 1.7) and participants with more than 5 to less than 6 hours of sleep (OR of 1.4) after controlling for compounding factors.

Previous studies have shown the association of severity of periodontal disease with poor sleep. ${ }^{1} 67$ Additionally, deep sleep deprivation is reported to worsen systemic health in rodent models showing increase of alveolar bone loss and gingival inflammation. ${ }^{6}$ However, a nationally representative study of participants who were aged 30 years analysed the relationship between periodontal disease and poor sleep, and the study did not show statistical significance. ${ }^{7}$ More recently, direct and independent association between sleep duration and the prevalence of periodontitis was found. ${ }^{8}$ Moreover, the association between tooth loss and obstructive sleep apnoea has 


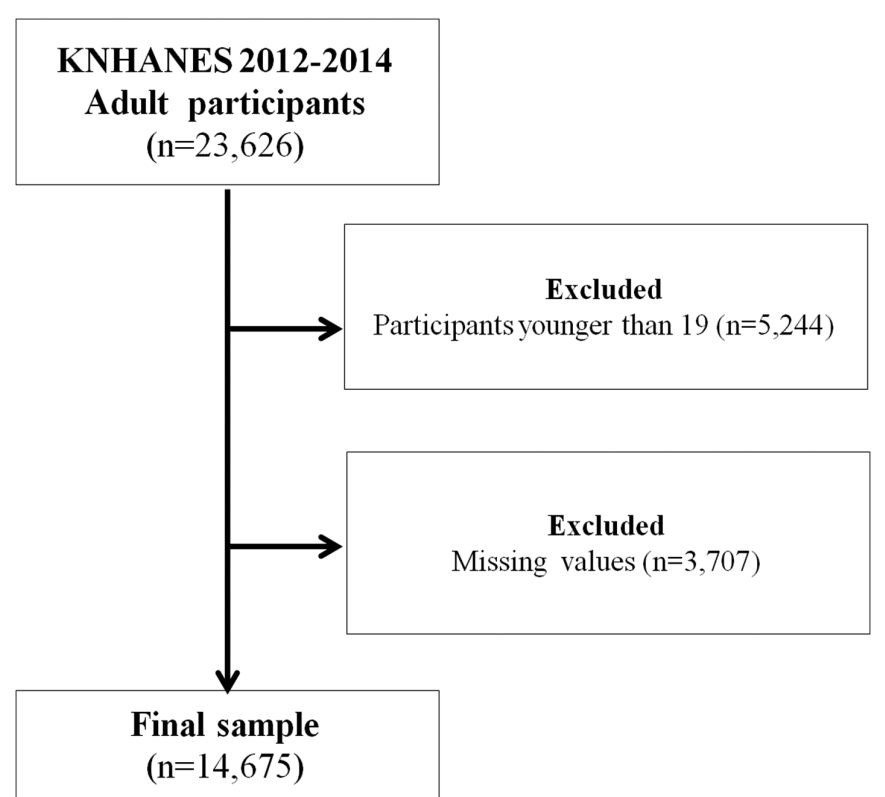

Figure 1 Participant flow chart. KNHANES, Korean National Health and Nutrition Examination Survey.

been evaluated in the previous studies. ${ }^{9}{ }^{10}$ Prevalence of high risk for obstructive sleep apnoea increased by $2 \%$ for each additional lost tooth and tooth loss was suggested to be an independent risk factor for obstructive sleep apnoea. ${ }^{10}$ However, the relationship between sleep duration and tooth loss has not been proven yet. It was hypothesised in this study that there is no significant association between short sleep duration and tooth loss. This study was performed to assess the association between sleep duration and tooth loss using nationally representative data.

\section{METHODS}

\section{Survey and subjects}

This study used data from the Korean National Health and Nutrition Examination Survey (KNHANES), which was conducted between 2012 and 2014 by the Division of Chronic Disease Surveillance under the Korean Centre for Disease Control and Prevention and the Korean Ministry of Health and Welfare. ${ }^{11-13}$ The KNHANES is a nationwide survey of non-institutionalised civilians that uses a stratified and multistage probability sampling design with a rolling survey sampling model. The sampling units were based on the population and housing consensus from the National Census Registry in Korea. Sample weights were used to calculate all statistics of this survey. The sample weights were created by considering survey non-response, the complex survey design and post-stratification to represent the Korean population with sample participants. Trained interviewers administered the standardised health examination and questionnaire. Initially, a total of 23626 individuals were candidates in the KNHANES survey. The analysis in this study was confined to a total of 18382 respondents over the course of 19 years $(\mathrm{n}=5233$ excluded). Finally, 14675 individuals whose responses contained no missing values for the outcome variables were analysed $(n=3707$ excluded, figure 1 and table 1).

All participants in the survey signed an informed consent prior to participation. This study was conducted according to the Helsinki Declaration-Based Ethical Principles for Medical Research Involving Human Subjects. This is a secondary data analysis, and the institutional reviewer board at Seoul St. Mary's Hospital, College of Medicine, The Catholic University of Korea, approved this study protocol (KC14EISI0636 and KC18ZESI0150). The analysis was performed after planning the research question.

\section{Patient and public involvement}

Neither patients nor public were involved in the development of the research question, in the analysis and in drawing conclusions from the results.

\section{Sociodemographic and lifestyle variables}

Participants' smoking status was categorised into two groups in accordance with respondents' answers on the self-report questionnaire: current smoker or not. Participants were categorised into the current drinker group by using the criterion of alcohol consumed within 1 month of the interview. ${ }^{14}$ The amount of pure alcohol consumed was calculated using the average number of alcoholic beverages consumed and the frequency of alcohol consumption based on the survey. Individuals were regarded as regular walkers if they walked at least five times per week for more than $30 \mathrm{~min}$ per session. Sleep duration was self-reported based on the survey. In this study, a short sleep was considered 5 hours or less; a long sleep was defined as 9 hours or more and the reference category was 6 to 8 hours. ${ }^{15}$

\section{Anthropometric and biochemical measurements}

Body weight and height were measured to the nearest $0.1 \mathrm{~kg}$ and $0.1 \mathrm{~cm}$, respectively, with participants in light indoor clothing and without shoes. Body mass index was calculated as body weight $(\mathrm{kg})$ divided by the squared height $\left(\mathrm{m}^{2}\right)$. Waist circumference was measured at the narrowest point between the lower border of the rib cage and the iliac crest while the participant was in a standing position. ${ }^{1617}$

A blood sample was collected from the antecubital vein of each participant after a fasting period of 8 hours. Concentrations of serum fasting plasma glucose, total cholesterol, triglycerides, high-density lipoprotein cholesterol and white cell count were measured from the sample. To measure the levels of serum fasting plasma glucose, total cholesterol, triglycerides and high-density lipoprotein cholesterol, an automated chemistry analyser (Hitachi Automatic Analyzer 7600, Hitachi, Tokyo, Japan) and commercially available kits (Daiichi, Tokyo, Japan) were used. ${ }^{18}$ 
Table 1 The baseline characteristics of the study individuals according to the number of natural teeth divided by 25

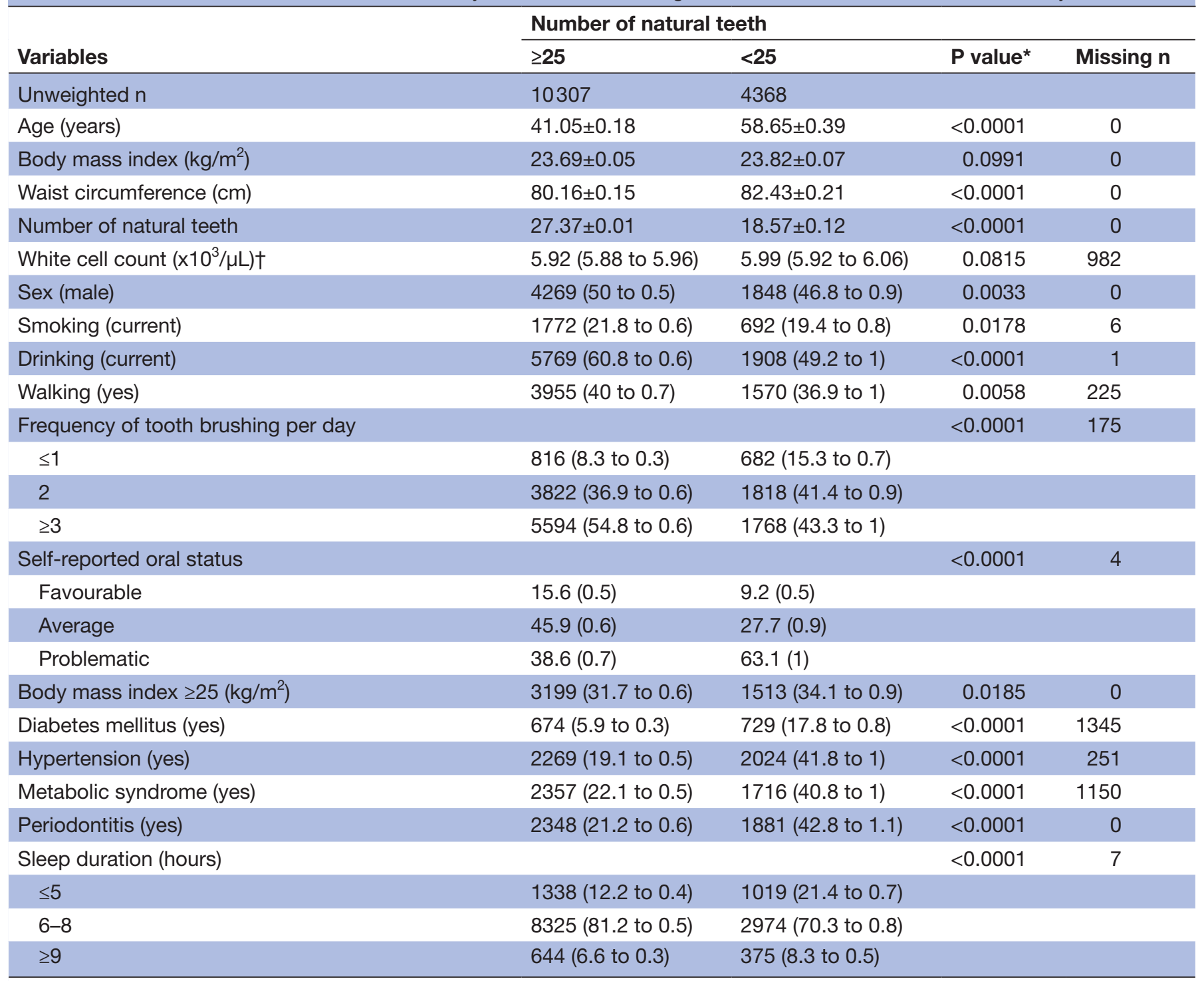

Data are presented as means $\pm \mathrm{SE}$ for continuous variables.

Data are presented as unweighted $\mathrm{n}$ (weighted percentage, SE) for categorical variables.

${ }^{*} \mathrm{P}$ values were obtained by independent $\mathrm{t}$-test for continuous variables or $\mathrm{X}^{2}$ test for categorical variables.

†Log transformation was applied to the value, and the geometric mean $(95 \% \mathrm{Cl})$ was shown.

Metabolic syndrome was determined if three or more of the following criteria were fulfilled ${ }^{19}$ : waist circumference $\geq 90 \mathrm{~cm}$ in men and $\geq 80 \mathrm{~cm}$ in women, fasting triglycerides $\geq 150 \mathrm{mg} / \mathrm{dL}$ or use of lipid-lowering medication, high-density lipoprotein cholesterol $<40 \mathrm{mg} / \mathrm{dL}$ in men and $<50 \mathrm{mg} / \mathrm{dL}$ in women or use of medication, blood pressure $\geq 130 / 85 \mathrm{~mm} \mathrm{Hg}$ or use of antihypertensive medication and, finally, fasting blood glucose $\geq 100 \mathrm{mg}$ / $\mathrm{dL}$ or current use of antidiabetes medication. Diabetes was diagnosed when fasting blood sugar was $>126 \mathrm{mg} / \mathrm{dL}$ or when the individual was currently using antidiabetic medications with diabetes as a component of the fasting glucose component of metabolic syndrome. ${ }^{20}$ Hypertension was defined as a systolic blood pressure of $>160 \mathrm{~mm}$ $\mathrm{Hg}$, a diastolic blood pressure of $>90 \mathrm{~mm} \mathrm{Hg}$ or the current use of systemic antihypertensive drugs. ${ }^{2122}$
Oral health behaviours, periodontal disease and the evaluation of the number of natural teeth

The time of day when participants brushed their teeth and used secondary oral products was recorded as oral health behaviours. ${ }^{23}$ We calculated the frequency of daily tooth brushing by the total number of times the teeth were brushed per day. Secondary oral products included the following: dental floss, mouthwash, interdental brushes, electric toothbrushes, irrigation devices, tongue cleaners, end-tufted brushes and a special device for dentures. Self-reported oral status was categorised into favourable, average and problematic. The presence of periodontal disease was evaluated using the WHO's community periodontal index. Periodontal disease was defined if the community periodontal index score was $\geq 3$. ${ }^{24}$ The status of natural teeth was categorised as 
28

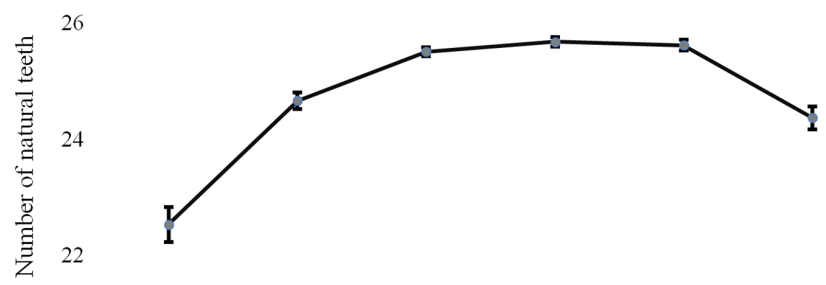

20
$4 \leq$
5
6
Sleep duration (hours)
$P$-value $<0.0001$

Figure 2 The number of natural teeth of the individuals according to sleep duration after adjustments for age, sex, smoking, drinking, walking, frequency of tooth brushing per day, body mass index and periodontitis.

primary tooth presence, permanent tooth presence, lack of tooth presence or permanent dental root fragment presence. In this study, the natural tooth was considered present if the status was among the first two categories and absent if the status was among the last two categories. We categorised the subjects' two groups according to the total number of natural teeth: $\geq 25$ teeth and $<25$ teeth. Trained and calibrated dentists examined the oral status of the participants, and training was provided to minimise the errors in the measurement by each examiner during the examination as a part of quality control.

\section{Statistical analyses}

All data are presented as mean \pm SE or unweighted $n$ (weighted percentage, SE). Logarithmic transformation was performed to achieve a normal distribution when necessary. Statistical analyses were performed using the survey procedure of the statistical software package (SAS V.9.2 for Windows, SAS Institute, Cary, North Carolina, USA) to account for the complex sampling design considering strata, cluster and weight. A $\mathrm{X}^{2}$ test for categorical variables or an independent $t$-test for continuous variables was performed to assess the differences in characteristics according to the number of natural teeth. A multivariable logistic regression analysis was used to evaluate the risk of tooth loss in relation to sleep duration, and OR and $95 \%$ CI were calculated after adjusting for potential confounders. Model 1 was adjusted for age and sex. In Model 2, adjustments were made for the variables in Model 1, plus smoking, drinking and walking habits and the frequency of tooth brushing. Model 3 was adjusted for the variables that were adjusted in Model 2, plus body mass index and periodontitis.

\section{RESULTS}

Figure 2 describes the number of natural teeth of the individuals according to sleep duration after adjustments for age; sex; smoking, drinking and walking habits; frequency of tooth brushing per day; body mass index and periodontitis $(p<0.05)$. Statistically significant differences were noted regarding the duration of sleep (hours) and tooth loss, showing a U-shaped association.

Table 1 shows the baseline characteristics of the study's individuals according to the number of natural teeth, which is standardised as 25. Significant differences were noted between the groups according to the participants' mean age; waist circumference; white cell count; smoking, drinking and walking habits; frequency of tooth brushing per day; self-reported oral status; body mass index $\geq 25$ $\left(\mathrm{kg} / \mathrm{m}^{2}\right)$; diabetes mellitus; hypertension; metabolic syndrome and periodontitis $(\mathrm{p}<0.05)$.

Table 2 shows the baseline characteristics of the study's individuals according to sleep duration. Participants with a sleep duration of 6-8hours showed the lowest prevalence of diabetes mellitus, hypertension, metabolic syndrome, periodontitis and meeting the waist circumference criteria for metabolic syndrome. The subgroup analysis categorised by age, sex, body mass index, diabetes mellitus, hypertension and metabolic syndrome (group 1: sleep duration $\leq 5$ hours, group 2: 6-8 hours and group $3: \geq 9$ hours) is shown in figure 3 . The association between short and long sleep duration and tooth loss was evident in both of the following groups: 19-64 years and $\geq 65$ years. The adjusted OR and their 95\% CI from multivariate logistic regression analyses for individuals with fewer than 25 natural teeth were significantly higher in short sleep and long sleep groups than in the group that slept 6-8hours with participants who had a body mass index $\leq 25$, without diabetes mellitus, without hypertension or without metabolic syndrome.

Table 3 shows the adjusted OR and their 95\% CI from multivariate logistic regression analyses for individuals with fewer than 25 natural teeth according to sleep duration. The adjusted OR and the $95 \%$ CI of the male individuals with fewer than 25 natural teeth were 1.426 (1.113 to 1.827 ), 1.290 (1.074 to 1.548$), 0.988$ (0.853 to 1.145$), 1$ (reference), 1.058 (0.907 to 1.235 ) and 1.620 (1.287 to 2.038) for sleep durations of 4 hours or less, 5 hours, 6 hours, 7 hours, 8 hours and 9 hours or more, respectively $(\mathrm{p}<0.05)$, after adjustments for age; sex; smoking, drinking and walking habits; frequency of tooth brushing per day; body mass index and periodontitis.

\section{DISCUSSION}

The aim of this study was to identify the associations between tooth loss and sleep duration. The results showed an increased OR of tooth loss with short and long sleep duration in adults at a statistically significant level.

Sleep problems have emerged as a significant medical and social concern. ${ }^{25}$ In general, younger people and women in the population reported more somatic and psychological complaints. ${ }^{26}$ In previous research, using a questionnaire, the overall prevalence of insomnia was $21.4 \%$ during the preceding month. ${ }^{27}$ The percentage of difficulty in initiating sleep was $8.3 \%$, the percentage 
Table 2 The baseline characteristics of the study individuals according to sleep duration

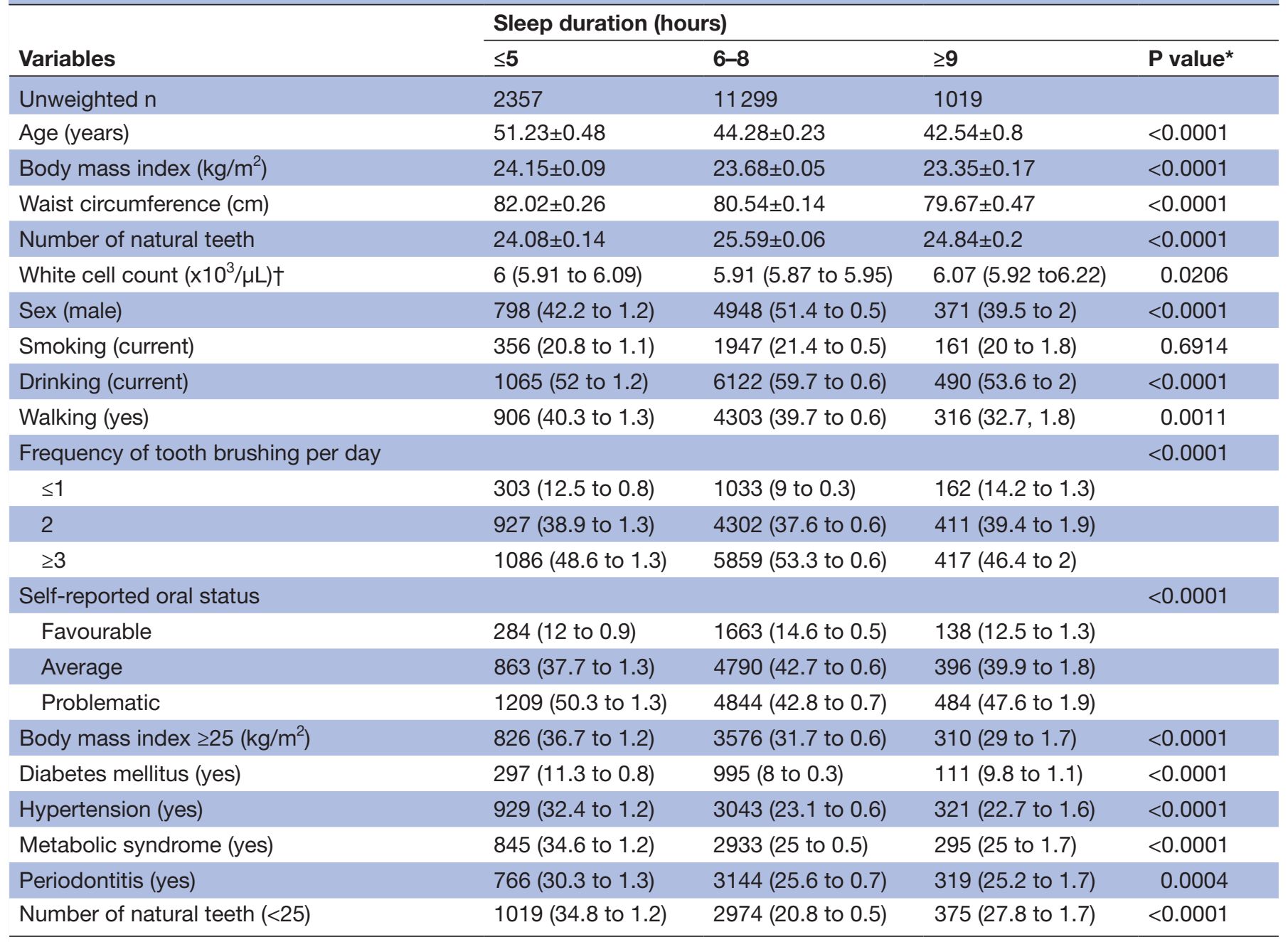

${ }^{*} \mathrm{P}$ values were obtained by independent $\mathrm{t}$-test for continuous variables or $\mathrm{X}^{2}$ test for categorical variables.

†Log transformation was applied to the value, and the geometric mean $(95 \% \mathrm{Cl})$ was shown.

of difficulty in maintaining sleep was $15.0 \%$ and the percentage of early morning awakening was $8.0 \%$. The overall prevalence of male and female insomnia was reported in another study. ${ }^{28}$ The percentage of male participants who had difficulty initiating sleep, difficulty maintaining sleep and poor perceived quality of sleep were $8.6 \%, 12.9 \%$ and $17.8 \%$, respectively. The percentage of female individuals who had difficulty initiating sleep, difficulty maintaining sleep and poor perceived quality of sleep were $12.6 \%, 16.2 \%$ and $20.2 \%$ respectively. It was also shown that $8 \%-18 \%$ of the general population reported sleep dissatisfaction. ${ }^{29}$

Previous studies continuously reported the association between health issues and sleep duration. ${ }^{43031}$ Higher total mortality was associated with both short and long sleep duration. ${ }^{30}{ }^{31}$ Similarly, both short and long sleep duration were associated with higher type 2 diabetes mellitus, cardiovascular disease, respiratory diseases and obesity. ${ }^{30}$ Previous studies suggested a U-shaped association between health outcomes and sleep duration. ${ }^{3031}$ Similarly, this study clearly showed that tooth loss associated with the duration of sleep (hours) presented a $\mathrm{U}$ shape after the adjustments of covariates. A subgroup analysis revealed that the U-shape association was maintained in the following groups: $19-64$ years and $\geq 65$ years. Moreover, subgroup analyses showed that short and long sleep duration were associated with higher incidence of tooth loss in participants without diabetes mellitus, those without hypertension and those without metabolic syndrome.

The definition of short and long sleep duration varies among different studies. ${ }^{15}$ 32-34 In previous studies, the reference category was defined as 7 hours, ${ }^{32} 7$ to 8 hours, ${ }^{35} 7$ to 9 hours, ${ }^{36} 6$ to 8 hours, ${ }^{37}$ and 9 hours. ${ }^{34}$ In this study, the reference was considered as 6 to 8 hours in the categorical evaluation.

This association between sleep duration and tooth loss may be explained by the following. Monocyte production of interleukin- 6 and tumour necrosis factor alpha was significantly higher in the morning after poor sleep, compared with the morning levels following uninterrupted sleep. ${ }^{38}$ Similarly, sleep deprivation induced a 


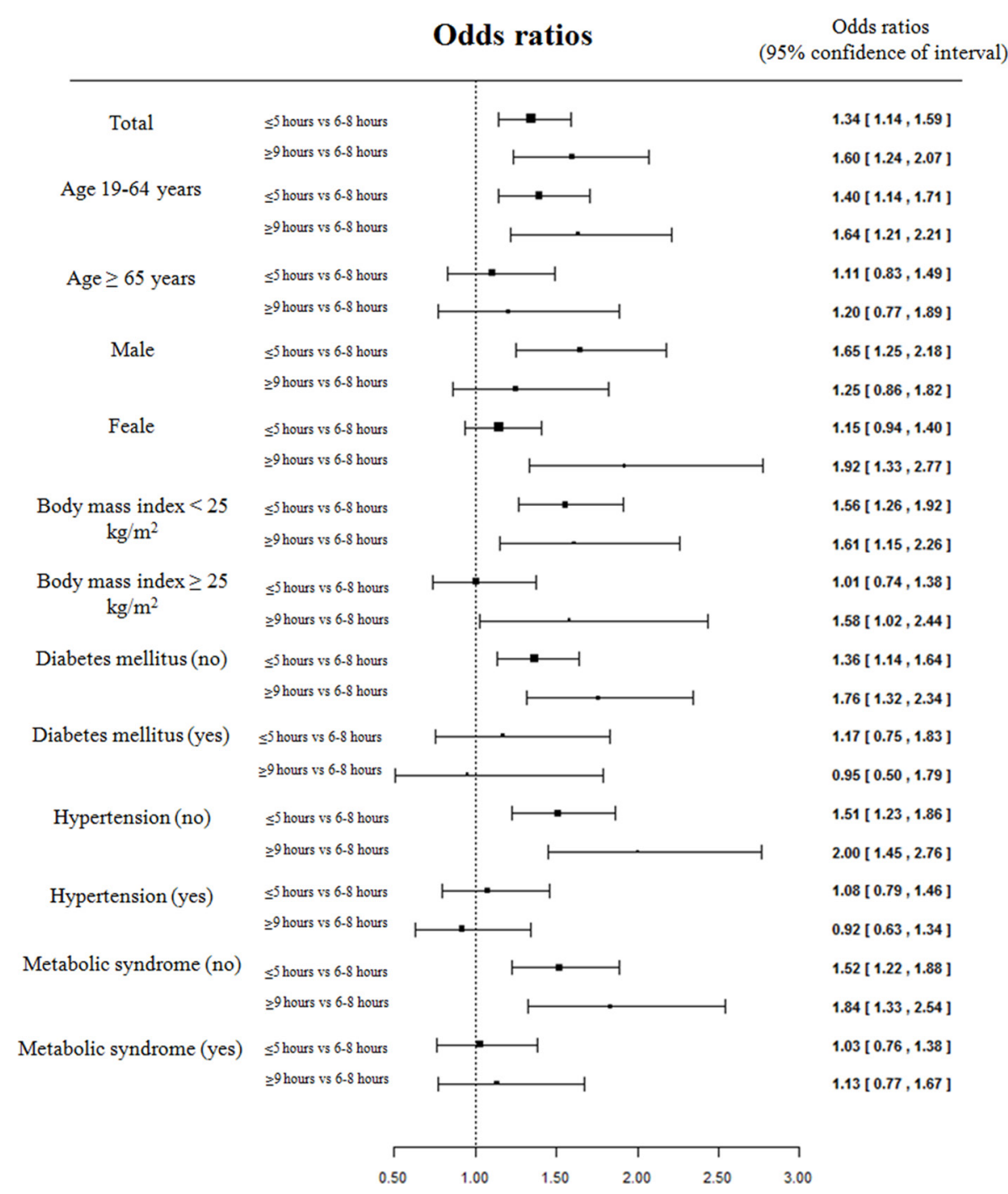

Figure 3 Subgroup analysis categorised by age, sex, body mass index, diabetes mellitus, hypertension and metabolic syndrome (group 1: sleep duration $\leq 5$ hours, group 2: $6-8$ hours and group 3: $\geq 9$ hours) as well as the adjusted OR and their $95 \% \mathrm{Cl}$ from multivariate logistic regression analyses for individuals with fewer than 25 natural teeth.

significant increase in E-selectin, intracellular adhesion molecule-1, interleukin-1 beta and anti-inflammatory cytokine interleukin-1 receptor antagonist; a significant decrease in $\mathrm{C}$ reactive protein and interleukin-6; and no significant change in vascular adhesion molecule- $1 .{ }^{39}$ It should also be considered that sociodemographic factors

Table 3 The adjusted OR and their $95 \% \mathrm{Cl}$ from multivariate logistic regression analyses for individuals $<25$ natural teeth according to sleep duration

\begin{tabular}{|c|c|c|c|}
\hline \multirow{2}{*}{$\begin{array}{l}\text { Variables } \\
\text { Sleep duration (hours) }\end{array}$} & \multicolumn{3}{|c|}{ Number of natural teeth $<25$} \\
\hline & Model 1 & Model 2 & Model 3 \\
\hline$\leq 4$ & 1.533 (1.23 to 1.91$)$ & 1.506 (1.203 to 1.884$)$ & $1.53(1.223$ to 1.915$)$ \\
\hline 5 & 1.295 (1.087 to 1.542$)$ & 1.264 (1.059 to 1.51$)$ & 1.274 (1.066 to 1.523$)$ \\
\hline 6 & 1.018 (0.884 to 1.172$)$ & 0.999 (0.867 to 1.152$)$ & 1.001 (0.868 to 1.155$)$ \\
\hline 7 & 1 (reference) & 1 (reference) & 1 (reference) \\
\hline 8 & 1.085 (0.936 to 1.257$)$ & $1.048(0.903$ to 1.215$)$ & 1.04 (0.897 to 1.206$)$ \\
\hline$\geq 9$ & 1.677 (1.345 to 2.09$)$ & $1.633(1.306$ to 2.041$)$ & 1.621 (1.298 to 2.024$)$ \\
\hline$P$ value & $<0.0001$ & $<0.0001$ & $<0.0001$ \\
\hline
\end{tabular}

Model 1: Age and sex adjusted.

Model 2: Model 1+smoking, drinking, walking and frequency of tooth brushing per day adjusted.

Model 3: Model 2+body mass index, and periodontitis adjusted. 
may be associated with sleep disorders. ${ }^{28}$ Among men, difficulty maintaining sleep and poor perceived quality of sleep were associated with never having married with the OR of 2.8 and 2.1, respectively. Difficulty maintaining sleep was also associated with being over 60 years old and/or divorced with OR of 2.7 and 3.7, respectively. Among women, difficulty maintaining sleep was associated with being widowed $(\mathrm{OR}=1.65)$ and unemployed $(\mathrm{OR}=1.60)$, and poor perceived quality of sleep was associated with advancing age $(\mathrm{OR}=0.63-0.50)$. The association of sleep deprivation with severity of periodontal disease was suggested in the previous study. ${ }^{1}$ Previous report suggested the association between the difficulty in sleep and the presence of dental caries. ${ }^{40}$ It is not rare that sleep problems are reported in individuals with headache and chronic orofacial pain. ${ }^{25}{ }^{41}$ Researchers suggested the need to refer patients with temporomandibular joint disorder complaining of sleep disturbance for polysomnographic evaluation, considering the high rates of primary insomnia and sleep apnoea. ${ }^{41}$ Another report showed that periodontitis prevalence was higher in individuals with long sleep duration. ${ }^{8}$ Interleukin-1 and tumour necrosis factor may be involved in tooth loss in participants with long sleep duration. ${ }^{42}$

The present study had several limitations to consider. The design of this study is cross-sectional, and the causal direction cannot be evaluated. ${ }^{43}$ The duration of sleep could be an influencing factor for tooth loss, but the opposite may be true. The duration of sleep was evaluated via interviews with the participants, and there may have been recall bias. ${ }^{44}$ It should also be considered that there may be residual confounding factors and there may be missing factors that affect sleep duration. Intrinsic need, sleep disorders, external environmental pressure, quality of sleep, naps and simple category in smoking state may also need to be considered. In spite of these limitations, this study used very well-organised and reliable data with complex, stratified and multistage clustered probability design. ${ }^{45}$ The dental examinations were performed by trained dentists. ${ }^{24}$ Collectively, these results can be considered as nationally representative data but limited to South Korean population.

\section{CONCLUSIONS}

Conclusively, the U-shaped association between sleep duration and tooth loss was suggested by multiple logistic regression analyses after adjusting for confounding factors. Moreover, subgroup analyses showed that short and long sleep duration were associated with greater tooth loss in participants without diabetes mellitus, those without hypertension and those without metabolic syndrome.

Acknowledgements A professional copyediting agency (Papercheck, Santa Barbara, California, USA) has checked the quality of the manuscript. The authors thank the Korea Centers for Disease Control and Prevention for providing the data.
Contributors $\mathrm{KH}$ and J-BP conceived experiments, analysed the data, wrote the manuscript, reviewed the manuscript and approved the final manuscript.

Funding This study was supported by Research Fund of Seoul St. Mary's Hospital, The Catholic University of Korea. This work was also supported by Basic Science Research Program through the National Research Foundation of Korea (NRF) funded by the Ministry of Science, ICT \& Future Planning (NRF-2017R1A1A1A05001307).

Competing interests None declared.

Patient consent Obtained.

Ethics approval This study was approved by institutional review board of Korean Center for Disease Control and Prevention (2012-01EXP-01-2C, 2013-07CON-03-4C, and 2013-12EXP-03-5C), and conducted according to the Helsinki Declaration based Ethical Principles for Medical Research Involving Human Subjects.

Provenance and peer review Not commissioned; externally peer reviewed.

Data sharing statement All data generated or analysed during this study are included in this article.

Open Access This is an Open Access article distributed in accordance with the Creative Commons Attribution Non Commercial (CC BY-NC 4.0) license, which permits others to distribute, remix, adapt, build upon this work non-commercially, and license their derivative works on different terms, provided the original work is properly cited and the use is non-commercial. See: http://creativecommons.org/ licenses/by-nc/4.0/

(C) Article author(s) (or their employer(s) unless otherwise stated in the text of the article) 2018. All rights reserved. No commercial use is permitted unless otherwise expressly granted.

\section{REFERENCES}

1. Grover V, Malhotra R, Kaur H. Exploring association between sleep deprivation and chronic periodontitis: A pilot study. J Indian Soc Periodontol 2015;19:304-7.

2. Wolk R, Gami AS, Garcia-Touchard A, et al. Sleep and cardiovascular disease. Curr Probl Cardiol 2005;30:625-62.

3. Cappuccio FP, D'Elia L, Strazzullo P, et al. Quantity and quality of sleep and incidence of type 2 diabetes: a systematic review and meta-analysis. Diabetes Care 2010;33:414-20.

4. Singh M, Drake CL, Roehrs T, et al. The association between obesity and short sleep duration: a population-based study. J Clin Sleep Med 2005;1:357-63.

5. Dørheim SK, Bondevik GT, Eberhard-Gran M, et al. Sleep and depression in postpartum women: a population-based study. Sleep 2009;32:847-55

6. Nakada T, Kato T, Numabe Y. Effects of fatigue from sleep deprivation on experimental periodontitis in rats. J Periodontal Res 2015;50:131-7.

7. Wiener RC. Relationship of Routine Inadequate Sleep Duration and Periodontitis in a Nationally Representative Sample. Sleep Disord 2016;2016:1-6.

8. Romandini M, Gioco G, Perfetti G, et al. The association between periodontitis and sleep duration. J Clin Periodontol 2017;44:490-501.

9. Bucca C, Cicolin A, Brussino L, et al. Tooth loss and obstructive sleep apnoea. Respir Res 2006;7:8.

10. Sanders AE, Akinkugbe AA, Slade GD, et al. Tooth loss and obstructive sleep apnea signs and symptoms in the US population. Sleep Breath 2016;20:1095-102.

11. Sorensen MD, Hsi RS, Chi T, et al. Dietary intake of fiber, fruit and vegetables decreases the risk of incident kidney stones in women: a Women's Health Initiative report. J Urol 2014;192:1694-9.

12. Lim SG, Han K, Kim HA, et al. Association between insulin resistance and periodontitis in Korean adults. J Clin Periodontol 2014;41:121-30.

13. Ko SH, Kwon HS, Kim DJ, et al. Higher prevalence and awareness, but lower control rate of hypertension in patients with diabetes than general population: the fifth korean national health and nutrition examination survey in 2011. Diabetes Metab J 2014;38:51-7.

14. Agarwal DP. Cardioprotective effects of light-moderate consumption of alcohol: a review of putative mechanisms. Alcohol Alcohol 2002;37:409-15.

15. Patel SR, Ayas NT, Malhotra MR, et al. A prospective study of sleep duration and mortality risk in women. Sleep 2004;27:440-4.

16. Weisell RC. Body mass index as an indicator of obesity. Asia Pac J Clin Nutr 2002;11(s8):S681-4. 
17. Oh SW, Shin SA, Yun YH, et al. Cut-off point of BMI and obesityrelated comorbidities and mortality in middle-aged Koreans. Obes Res 2004;12:2031-40.

18. Wallace TM, Levy JC, Matthews DR. Use and abuse of HOMA modeling. Diabetes Care 2004;27:1487-95.

19. Alberti KG, Eckel RH, Grundy SM, et al. Harmonizing the metabolic syndrome: a joint interim statement of the International Diabetes Federation Task Force on Epidemiology and Prevention; National Heart, Lung, and Blood Institute; American Heart Association; World Heart Federation; International Atherosclerosis Society; and International Association for the Study of Obesity. Circulation 2009;120:1640-5.

20. Jeon JY, Ko SH, Kwon HS, et al. Prevalence of diabetes and prediabetes according to fasting plasma glucose and $\mathrm{HbA} 1 \mathrm{c}$. Diabetes Metab J 2013;37:349-57.

21. Weber MA, Julius S, Kjeldsen SE, et al. Blood pressure dependent and independent effects of antihypertensive treatment on clinical events in the VALUE Trial. Lancet 2004;363:2049-51.

22. Lenfant $\mathrm{C}$, Chobanian $\mathrm{AV}$, Jones DW, et al. Seventh report of the Joint National Committee on the Prevention, Detection, Evaluation, and Treatment of High Blood Pressure (JNC 7): resetting the hypertension sails. Hypertension 2003;41:1178-9.

23. Kim YH, Kim DH, Lim KS, et al. Oral health behaviors and metabolic syndrome: the 2008-2010 Korean National Health and Nutrition Examination Survey. Clin Oral Investig 2013.

24. Park JB, Han K, Park YG, et al. Association between alcohol consumption and periodontal disease: the 2008 to 2010 Korea National Health and Nutrition Examination Survey. J Periodontol 2014;85:1521-8.

25. Kato T, Yamaguchi T, Okura K, et al. Sleep less and bite more: sleep disorders associated with occlusal loads during sleep. J Prosthodont Res 2013;57:69-81.

26. Kim K, Uchiyama M, Liu X, et al. Somatic and psychological complaints and their correlates with insomnia in the Japanese general population. Psychosom Med 2001;63:441-6.

27. Kim K, Uchiyama M, Okawa M, et al. An epidemiological study of insomnia among the Japanese general population. Sleep 2000;23:1-7.

28. Doi Y, Minowa M, Okawa M, et al. Prevalence of sleep disturbance and hypnotic medication use in relation to sociodemographic factors in the general Japanese adult population. J Epidemiol 2000;10:79-86.

29. Ohayon MM. Epidemiology of insomnia: what we know and what we still need to learn. Sleep Med Rev 2002;6:97-111.

30. Stranges S, Dorn JM, Shipley MJ, et al. Correlates of short and long sleep duration: a cross-cultural comparison between the United Kingdom and the United States: the Whitehall II Study and the Western New York Health Study. Am J Epidemiol 2008;168:1353-64.

31. Knutson KL, Turek FW. The U-shaped association between sleep and health: the 2 peaks do not mean the same thing. Sleep 2006;29:878-9.

32. Mallon L, Broman JE, Hetta J. Sleep complaints predict coronary artery disease mortality in males: a 12-year follow-up study of a middle-aged Swedish population. J Intern Med 2002;251:207-16.

33. Lan TY, Lan TH, Wen CP, et al. Nighttime sleep, Chinese afternoon nap, and mortality in the elderly. Sleep 2007;30:1105-10.

34. Gale C, Martyn C. Larks and owls and health, wealth, and wisdom. BMJ 1998;317:1675-7.

35. Hublin C, Partinen M, Koskenvuo M, et al. Sleep and mortality: a population-based 22-year follow-up study. Sleep 2007;30:1245-53.

36. Kojima M, Wakai K, Kawamura T, et al. Sleep patterns and total mortality: a 12-year follow-up study in Japan. J Epidemio 2000;10:87-93.

37. Stone KL, Ewing SK, Ancoli-Israel S, et al. Self-reported sleep and nap habits and risk of mortality in a large cohort of older women. $J$ Am Geriatr Soc 2009;57:604-11.

38. Irwin MR, Wang M, Campomayor CO, et al. Sleep deprivation and activation of morning levels of cellular and genomic markers of inflammation. Arch Intern Med 2006;166:1756-62.

39. Frey DJ, Fleshner M, Wright KP. The effects of 40 hours of total sleep deprivation on inflammatory markers in healthy young adults. Brain Behav Immun 2007;21:1050-7.

40. Shantinath SD, Breiger D, Williams BJ, et al. The relationship of sleep problems and sleep-associated feeding to nursing caries. Pediatr Dent 1996;18:375-8.

41. Smith MT, Wickwire EM, Grace EG, et al. Sleep disorders and their association with laboratory pain sensitivity in temporomandibular joint disorder. Sleep 2009;32:779-90.

42. Jewett KA, Krueger JM. Humoral sleep regulation; interleukin-1 and tumor necrosis factor. Vitam Horm 2012;89:241-57.

43. Han K, Hwang E, Park JB. Excessive consumption of green tea as a risk factor for periodontal disease among Korean adults. Nutrients 2016;8:8.

44. Han K, Ko Y, Park YG, et al. Associations between the number of natural teeth in postmenopausal women and duration of lactation: the 2010-2012 Korea National Health and Nutrition Examination Survey. Maturitas 2016;85:73-8.

45. Han K, Ko Y, Park YG, et al. Associations between the periodontal disease in women before menopause and menstrual cycle irregularity: the 2010-2012 Korea National Health and Nutrition Examination Survey. Medicine 2016;95:e2791. 